France. Seizures occurred during sleep and were preceded by a short period of arousal. A brief symmetrical flattening of the EEG, associated with apnea, tachycardia, and tonic motor activity, was followed by a long (1-2 minute) bilateral discharge of spikes and sharp waves, simultaneous with vocalizations, chewing, and focal or generalized clonic activity, often asymmetrical and asynchronous. EEG and clinical symptoms varied from left to right from one seizure to the next, and a stable focus was never observed. (Hirsch E et al. Electroclinical signs of benign neonatal familial convulsions. Ann Neurol Dec 1993; 34:835-841). (Respond: Dr Hirsch, Service d'Epileptologie Clinique, Clinique Neurologique, Hopitaux Universitaires de Strasbourg, 1 place de l'Hopital, 67091 Strasbourg Cedex, France).

COMMENT. BNFCs are brief seizures beginning at the second or third day after birth and occurring for several days or weeks. Less than $10 \%$ have seizures in later childhood and adulthood. The gene for this syndrome has been localized to the long arm of chromosome 20, but BNFCs may be genetically heterogeneous. The pattern of the seizures is similar to that of generalized tonic-clonic epilepsy, in which the initial tonic phase may be asymmetrical. The asymmetry of BNFCs may be explained by immaturity of the corpus callosum in the neonate. The interictal EEG is either normal or shows bursts of sharp alternating theta rhythms, a theta pointu alternant pattern. Frequent BNFCs have no postictal deficit or exhaustion and no adverse effect on development of the child.

\title{
GENETICS OF UNVERRICHT-LUNDBORG MYOCLONUS EPILEPSY
}

Clinical and molecular genetic data on a family from the United States in which four of five sibs were affected with progressive myoclonus epilepsy of Unverricht-Lundborg type are reported from the University of Helsinki, Finland; National Cancer Institute, Bethesda, MD; and the VA Hospital, University of Florida, Gainesville, FL. Onset was between 9 and 11 years with grand mal seizures. Progressive stimulus-sensitive myoclonus developed at 11 to 13 years of age. Generalized spike-wave and polyspike-wave paroxysms and photo-sensitivity were characteristic EEG findings. The gene (EPM1) has been mapped to chromosome 21 in Finland, and the gene in this non-Finnish American family was also linked to the EPM1 region. Crossover events refined the gene localization between loci CBS and D21S112, leading to greater accuracy of genetic prediction based on linkage analysis. (Lehesjoki A-E et al. Progressive myoclonus epilepsy of Unverricht-Lundborg type: A clinical and molecular genetic study of a family from the United States with four affected sibs. Neurology Nov 1993; $\underline{3}$ :2384-2386). (Reprints: Dr Anna-Elina Lehesjoki, Dept Med Genetics, PO Box 21 (Haartmanin-katu 3), 00014 University of Helsinki, Finland). 
COMMENT. Causes of progressive myoclonus epilepsy include Unverricht-Lundborg disease (ULD), Lafora's disease, MERRF, Kufs' neuronal ceroid lipofuscinosis, and sialidoses. ULD is relatively common in Finland. Cochius JI et al, at the Montreal General Hospital, have performed linkage analysis in 8 families, including 4 of neither Baltic nor Mediterranean origin. They report that ULD diagnosed in areas remote from the Baltic maps to the same locus on chromosome 21 as the Finnish form, and suggest that all ULD, regardless of geographic origin,

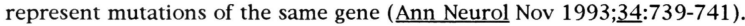
Lafora's disease, characterized by intraneuronal inclusions, is a form of myoclonus epilepsy not linked to EPM1 and chromosome 21. The recent advances in molecular genetics of neurological disease are reviewed in two current journals: Rosenberg RN et al. Arch Neurol Nov 1993; $\underline{50: 1123-}$ 1265, and Martin JB. Ann Neurol Dec 1993; $\underline{34: 757-773 . ~}$

\section{TRAUMATIC DISORDERS}

\section{SHAKEN BABY SYNDROME}

Non-accidental head injury, with particular reference to whiplash shaking injury, is reviewed by paediatric neurologists at the Royal Hospital for Sick Children, Edinburgh. Of 30 children with non-accidental head injury followed by the authors, 17 were due to shaking and 13 to impact. Subdural hematoma was diagnosed in 16 (53\%), subarachnoid hemorrhage in 3, and intracerebral hemorrhage in 4 . Eleven (37\%) developed post-hemorrhagic hydrocephalus. Velocity, the number of shakes per second, and duration are important components of degree of injury. The most common age for whiplash abuse is 5 months, when the head is large in relation to the body, and the neck muscles and head control are weak. Shaking injuries are rare after the second year. Vascular, midbrain, and white matter shearing injuries, cerebral edema, and retinal and subdural hemorrhages result. The clinical diagnosis and medico-legal aspects of non-accidental head injury are discussed in detail. (Brown JK, Minns RA. Non-accidental head injury, with particular reference to whiplash shaking injury and medico-legal aspects. Dev Med Child Neurol Oct 1993; $35: 849-869)$. (Respond: Dr JK Brown, Royal Hospital for Sick Children, Sciennes Road, Edinburgh EH9 1LF, Scotland).

COMMENT. Despite an increase in support services and media publicity, non-accidental head injury remains the leading cause of death or long-term disability among child abuse cases in Scotland and in the USA. The American Academy of Pediatrics Committee on Child Abuse and Neglect review the "Shaken baby syndrome: Inflicted cerebral trauma" in the current issue of Pediatrics Dec 1993;92:872-875. Subtle or mild trauma is particularly difficult to diagnose, often mistaken for viral illness, feeding problems, or infant colic. 